\title{
Session 5: Molecular biology (II)
}

\author{
Tuesday 17 September 2002, Moderators: O.R. Burrone and R. Burioni
}

[14.00-14.20]

\section{DeImmunised immunotoxins for multiple targets Frank Carr \\ Biovation Ltd, Aberdeen Science Park, Aberdeen, UK}

The large output of multiple disease-related target proteins from oncology-related genomics and proteomics initiatives demands enhanced capabilities in tools to validate and functionalise these targets and, for certain targets thereafter, improved capabilities for generation of therapeutic antibodies to utilise these targets and to use such antibodies to eradicate disease. In oncology applications, principal enhancements required are;

(1) technologies to rapidly isolate antibodies with suitable affinities and specificities to validate targets

(2) technologies to ensure antibodies are not immunogenic in patients

(3) technologies to ensure that the resultant antibodies can kill tumour cells

To address these requirements, Biovation is establishing a suite of technologies to rapidly generate monoclonal antibodies to new targets and to generate therapeutic derivatives of such antibodies fused to toxins ("DeImmunised immunotoxins").

These technologies are as follows;

(1) rapid isolation of antibodies - this technology combines immunisation of BalbC mice with the generation and rapid screening of phageantibody libraries that comprehensively sample the mouse immuno-repertoire.

(2) DeImmunisation - human helper T cell epitopes within lead antibodies from (1) are identified and eliminated by amino acid substitutions within the variable region. Human constant regions are added to produce "DeImmunised" antibodies deficient in the ability to trigger T cell-dependant immunogenicity in vivo.
(3) non-immunogenic toxin - for fusion to antitumour targeted DeImmunised antibodies, a plant molecule has been "DeImmunised" to remove human helper $\mathrm{T}$ cell epitopes with the objective of rendering the toxin non-immunogenic in patients.

The basis and validation of these technologies will be described together with data illustrating the potential utility of the technology suite in treatment of cancer.

\section{[14.20-14.40]}

\section{From EST to IHC - Human antibodies for target validation and drug development Harald Watzka \\ International Project Management, MorphoSys AG, Lena-Christ-Strasse 48, D-82152 \\ Martinsried/Planegg, Germany}

MorphoSys' Human Combinatorial Antibody Library $\left(\mathrm{HuCAL}{ }^{\mathrm{B}}\right)$ ) has been proven to be a rich source of high-quality human antibodies selected against very different and complex antigens. The recently released new proprietary antibody library, HuCAL ${ }^{B}$ GOLD contains several significant innovations over previous versions of the HuCAL ${ }^{\circledR}$ library and is combined with the Company's proprietary CysDisplay ${ }^{\mathrm{TM}}$ screening technology. Importantly, the new library possesses diverse repertoires in all six CDR regions of the underlying antibody genes. Moreover in combination with the CysDisplay $^{\mathrm{TM}}$ screening technology it is expected that the recovery of high affinity antibodies will be facilitated. As such, the new library and screening system is intended to be a cornerstone of a future Company product development program.

The overall robustness of our technology allowed us to develop a high-throughput antibody generation program (AutoCAL ${ }^{\mathrm{TM}}$ ), which puts antibody generation on the same high-throughput footing as many other modern discovery processes, from gene sequencing to 
drug screening. Combined with our anti-EST encoded polypeptide antibody approach and the preferred access to one of the world's largest human tissue banks, HuCAL ${ }^{\circledR}$ antibodies are excellent tools for characterization and validation of new therapeutic targets. The application of selected antibodies for target validation and for pre-clinical studies will be discussed.

\section{[14.40-15.00]}

\section{Selecting and optimising therapeutic antibody leads \\ Celia P. Shorrock and Lutz Jermutus \\ Cambridge Antibody Technology, Melbourn, \\ Cambridgeshire, $U K$}

Both phage display and ribosome display systems can be used effectively to isolate large numbers of sequence-diverse antibodies from nave libraries. Once leads have been identified the same display technologies can be used to optimize the characteristics of the drug candidates. Using case studies we will demonstrate the integration of display-based selections, highthroughput screening and secondary cell-based assays to develop therapeutic antibodies, and will use these examples to highlight the advantages specific to the different types of display system.

[15.00-15.20]

Troybodies $^{\mathrm{TM}}$, a novel approach to cancer vaccines Ole Henrik Brekke ${ }^{\mathrm{a}}$, Inger Sandlie ${ }^{\mathrm{b}}$ and Bjarne Bogen $^{\mathrm{b}}$

${ }^{a}$ Department New Technologies, Affitech AS, Oslo, Norway

${ }^{\mathrm{b}}$ University of Oslo, Norway

Troybodies $^{\mathrm{TM}}$ is a new cellular vaccine technology with the potential application within the treatment of cancer and infectious diseases.

Endocytosed proteins are processed in antigen presenting cells (APCs) to create short peptides that are presented by MHC classII molecules to CD4 positive
T-cells. The mechanism of such T-cell epitope presentation is exploited by the Troybody ${ }^{\mathrm{TM}}$ concept.

Troybodies $^{\mathrm{TM}}$ are genetically engineered recombinant human antibodies in which specific T-cell epitopes, i.e. peptides, are inserted in the loops of the immunoglobulin constant regions. We have successfully inserted different T-cell epitopes in different loops of constant domains in the human IgG3 molecule. The V-regions show specificity to APCs allowing the efficient targeting, internalisation and processing of Troybodies $^{\mathrm{TM}}$. Troybodies ${ }^{\mathrm{TM}}$ are able to stimulate specific T-cell activation both in vitro and in vivo. Recent developments within the Troybody ${ }^{\mathrm{TM}}$ technology will be discussed.

[15.20-15.40]

Affinity maturation of human Fab antibodies by yeast display

Simon E. Hufton

Dyax S.A., Belgium

The rapid and efficient affinity maturation of biomolecules is a critical component of the drug development pipeline. Selection of yeast displayed repertoires by fluorescent activated cell sorting has proven an effective method for the affinity maturation of $\mathrm{scFv}$ antibodies. We will present for the first time the display and affinity maturation of human Fab antibodies by similar selection of yeast displayed repertoires.

As Fab antibodies are composed of discrete light chain (LC) and heavy chain (HC) domains we have used yeast mating of separate LC and $\mathrm{HC}$ repertoires to generate combinatorially diverse repertoires. Repertoires generated by this procedure are in excess of $5 \times 10^{9}$ independent clones and represent diversity which has previously been regarded as beyond yeast using conventional chemical transformation. The application of this technology to the affinity maturation of Fab antibodies will be presented. Furthermore we will discuss the potential of this technology for the generation of large nave Fab antibody repertoires. 